\title{
Gastric Mixed
}

\section{Adenoneuroendocrine}

Carcinoma with a Trilineage Cell

Differentiation: Case Report and Review of the Literature

\author{
Marinos Pericleous ${ }^{a}$ Christos Toumpanakis ${ }^{a}$ \\ Heather Lumgair ${ }^{a}$ Martyn E. Caplin ${ }^{a}$ \\ Luke Morgan-Rowe $^{\mathrm{b}} \quad$ Ian Clark $^{\mathrm{c}}$ Tu Vinh Luong \\ Departments of ${ }^{a}$ Neuroendocrine Tumours, European Center of Excellence, \\ ${ }^{b}$ Radiology and ${ }^{\mathrm{C}}$ Pathology, Royal Free Hospital, London, UK
}

Key Words

MANEC - Gastric neuroendocrine tumours - Trilineage differentiation

\begin{abstract}
Most gastric neuroendocrine tumours are well differentiated and considered as neuroendocrine neoplasms, whilst poorly differentiated lesions are considered as neuroendocrine carcinomas and account for only $6-16 \%$ of gastric neuroendocrine tumours. Gastric mixed adenoneuroendocrine carcinomas are rare malignancies usually composed of a neuroendocrine carcinoma and an adenocarcinoma with a variable grade of differentiation. Here, we report an unusual and rare gastric mixed adenoneuroendocrine carcinoma with a trilineage cell differentiation including a neuroendocrine carcinoma, an adenocarcinoma and a squamous cell carcinoma. A brief discussion of the histopathological features, biological behaviour and treatment of this rare tumour type is presented.
\end{abstract}

\section{Introduction}

Gastric neuroendocrine tumours (NETs) are usually well differentiated, nonfunctioning neoplasms consisting of enterochromaffin-like (ECL) cells. Three distinct tumour types are recognised: (1) type I (74\% of gastric NETs), associated with autoimmune chronic atrophic gastritis (A-CAG); (2) type II (6\% of gastric NETs), associated with multiple endocrine neoplasia type 1 (MEN1) and Zollinger-Ellison syndrome (ZES), and (3) type III, sporadic (13\% of gastric NETs), not associated with 
A-CAG or MEN1/ZES. Of the three types of ECL cell NETs, type I and type II tumours are invariably associated with ECL cell hyperplasia [1] and hypergastrinaemia, resulting from a secondary response to antral gastrin cells to achlorydria in type I and from unregulated hormone release by a gastrinoma in type II. Type III tumours are sporadic, not associated with hypergastrinaemia or ECL cell hyperplasia. They are usually single lesions and often display more aggressive features than type I and type II tumours. Due to the difference in behaviour between the three types of NETs, type I and type II tumours are considered as neuroendocrine neoplasms, whilst type III tumours are mostly neuroendocrine carcinomas (NECs).

Recently, to include all poorly differentiated gastric NETs which consist of other than ECL gastric endocrine cells (serotonin or gastrin producing) and also a very rare type of gastric malignancies, the mixed adenoneuroendocrine carcinomas (MANECs), another type, 'type IV gastric NET', has been suggested.

Due to their rarity, our knowledge of gastric MANECs is limited and mainly based on a small series of patients and case reports. MANECs are a special type of tumour, characterised by an intimate mixture of two histologically different tumours, a NEC and a gland-forming carcinoma (adenocarcinoma). Both components are malignant.

Here, we report an interesting case of a gastric MANEC composed of a NEC and an exocrine component with two different cell differentiations including a moderately differentiated adenocarcinoma and a squamous cell carcinoma, of which only the NEC component has metastasised to the liver.

\section{Case Description}

We report the case of an 81-year-old man who presented 4 months earlier to his local hospital with right hypochondrial pain, palpitations, diarrhoea up to 10 times daily, nausea, anorexia and weight loss. His past medical history included severe ischaemic cardiomyopathy and diabetes mellitus type II. A noncontrast CT scan of his abdomen revealed multiple liver lesions. An ultrasound-guided fine needle aspiration of one of these lesions was reported as 'metastasis from NET'. Common tumour markers (CEA, CA 19-9) were not elevated. An 18F-FDG-PET scan revealed hypermetabolic areas in the liver, para-oesophageal, porta hepatis and para-aortic regions. The patient was referred to our hospital for further medical management.

Upon arrival, he was treated aggressively for acute kidney injury due to dehydration and underwent a noncontrast staging CT scan of his chest, abdomen and pelvis. The scan showed a gastric tumour with hepatic, intra-abdominal and left supraclavicular lymph node metastases. No pulmonary metastases were observed.

For further evaluation of the gastric lesion, the patient went on to have oesophagogastroduodenoscopy which revealed a large ulcerating malignant-looking mass in the antrum of the stomach (fig. 1), gastritis and duodenitis. The histology from the gastric mass showed a 'MANEC with a small area of squamous differentiation'. Neuroendocrine markers for chromogranin and synaptophysin were positive in the neuroendocrine component only and negative in the exocrine component of both adenocarcinoma and squamous cell carcinoma (fig. $2 \mathrm{a}-\mathrm{f}$ ). The tumour mitotic index was 52 mitoses per 10 high-power fields, and the Ki67 was 65\%. Further biochemical and functional imaging work-up was carried out. The serum level of chromogranin A was $551 \mathrm{pmol} / \mathrm{l}$ (normal level $<60 \mathrm{pmol} / \mathrm{l}$ ), and the urine 5-HIAA level was $30 \mu \mathrm{mol} /$ day (normal level 0-42 $\mu \mathrm{mol} /$ day).

In light of the neuroendocrine component, the patient underwent Indium-111 pentetreotide scinitgraphy (Octreoscan ${ }^{\mathrm{TM}}$ ), which showed avid uptake in the hepatic lesions, stomach and in a pararenal lymph node (ig. 3 ). On the basis of tumour biology and the disease extent, the patient would 
have been a candidate for cytotoxic chemotherapy. However, due to his other comorbidities and poor performance status, he was commenced only on long-acting somatostatin analogues and best supportive/palliative treatment. Following this, the patient's gastrointestinal symptoms partially improved and he was discharged back home.

\section{Discussion}

Gastrointestinal tumours displaying both exocrine and neuroendocrine differentiation are well recognised, although uncommon. Most of them arise in the appendix, but they also occur in the colon, stomach, oesophagus, duodenum and gallbladder. The association of the two components can vary in a spectrum ranging from nonendocrine neoplasms - either adenomas or carcinomas - with interspersed neuroendocrine cells to truly composite/mixed tumours showing an intimate mixture of neuroendocrine and exocrine tumour aggregates and to collision tumours in which the neuroendocrine neoplasm/carcinoma and the adenoma/adenocarcinoma are closely juxtaposed but not admixed [2]. Fujiyoshi et al. [3] reclassified mixed endocrine and nonendocrine epithelial tumours by dividing the tumours into six categories: (1) neuroendocrine cells interspersed within carcinomas; (2) carcinoids (NETs) with interspersed nonendocrine cells; (3) composite glandular-neuroendocrine cell carcinomas containing areas of carcinoid and conventional carcinomas; (4) collision tumours in which NETs and conventional carcinomas are closely juxtaposed but not admixed; (5) amphicrine tumours predominantly composed of cells exhibiting concurrent neuroendocrine and nonendocrine differentiation, and (6) combinations of the previous types. According to the World Health Organisation classification of tumours of the digestive system, tumours can be classified as truly mixed only if both components are present in significant proportions - at least $30 \%$ of either component should be identified to qualify for this definition [4]. In 2010, mixed exocrine-NECs have been renamed as MANECs by the World Health Organisation.

Gastric MANECs are rare and usually described to be composed of an exocrine component represented by an adenocarcinoma with a variable grade of differentiation and a neuroendocrine component usually represented by a NEC and rarely a NET. The case reported here is a peculiar MANEC, displaying a trilineage cell differentiation including a NEC, an adenocarcinoma and a squamous cell carcinoma (fig. 2a,b). The three components are intimately intermingled, although all three phenotypes are well recognisable as three distinct histological tumours with three different immunophenotypes (fig. 2c-f). A very few cases of MANECs with a squamous cell carcinoma component have been previously described in the literature [5-7]. Due to their rarity, very little is known about their histogenesis. Interesting findings have been published by Furlan et al. [8], including 5 mixed endocrine-exocrine tumours (2 gastric and 3 colonic) and 1 rectal collision tumour studied using 35 polymorphic microsatellite markers. A close genetic relationship between the two distinct histologic components within mixed endocrine-exocrine tumours was found, supporting the hypothesis that a monoclonal mechanism of tumorigenesis was the most frequent genetic event in mixed exocrine-endocrine tumours. Clonal divergence was observed in the only collision tumour investigated, composed of a well differentiated endocrine carcinoma associated with an adenocarcinoma, confirming the existence of double tumours growing next to each other coincidentally but showing different histogenesis and different tumorgenetic pathways. Based on the findings of Furlan et al. [8], we can 
postulate that our gastric MANEC, composed of three histologically distinct tumours but intimately mixed together, is most likely derived from a common pluripotent progenitor/stem cell. A few other authors have postulated the origin from a pluripotential precursor cell $[6,7,9]$.

As for the histogenesis, the natural history of the gastric MANECs is also still unclear. In our case, the clinical behaviour seemed to depend on the NEC component. The fine needle aspiration of one of the liver lesions had demonstrated the neuroendocrine nature of the liver metastases, lately confirmed by the avid uptake on Octreoscan. Kim and Chae [10] have recently published similar findings: the NEC component of their gastric MANEC had shown more aggressive features, invading into the serosa and the lymphatic glands, whereas the glandular carcinoma was confined to the mucosa and submucosa. Volante et al. [11] reported that the clinical behaviour of composite carcinomas depends on the adenocarcinomatous component if the associated endocrine component is well differentiated and upon the neuroendocrine component if it is poorly differentiated.

Treatment of localized gastric NECs usually involves total gastrectomy. In cases with distant metastases, combination of cisplatin and etoposide has been recommended [12]. Ideally, we would have commenced our patient on a platinum-based chemotherapy regimen. However, in view of the patient's poor performance status, we started him on palliative treatment with long-acting somatostatin analogues. The patient improved and was able to be discharged home.

In summary, we reported a peculiar gastric MANEC with trilineage cell differentiation (NEC + adenocarcinoma + squamous cell carcinoma), of which only the NEC component metastasised to the liver. As all MANECs seem to be very aggressive from the early beginning and platinum-based chemotherapy should be the first-choice treatment in advanced disease, there are no data to support the hypothesis that the presence of a third cell clone can have some implications to the overall behaviour of those tumours. 


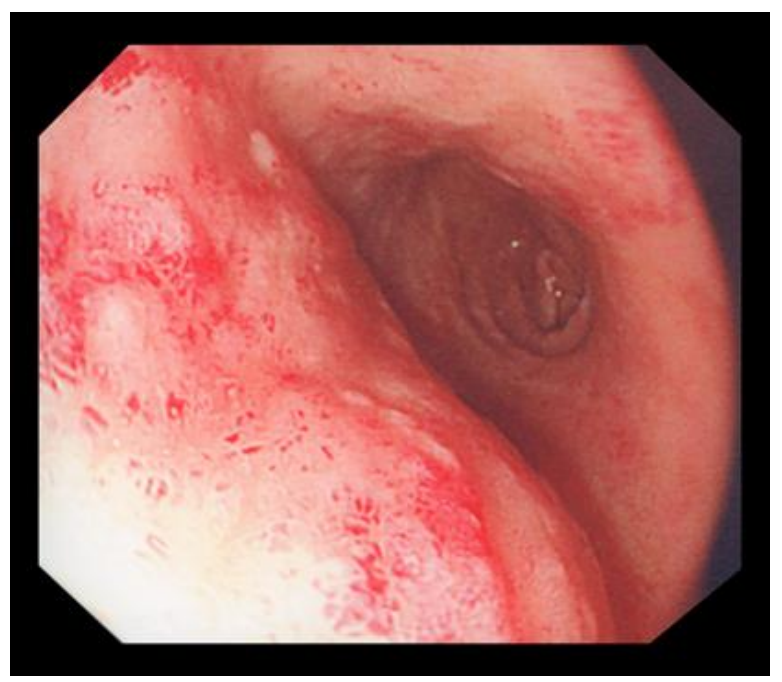

Fig. 1. Macroscopic appearance of the tumour in the stomach. 

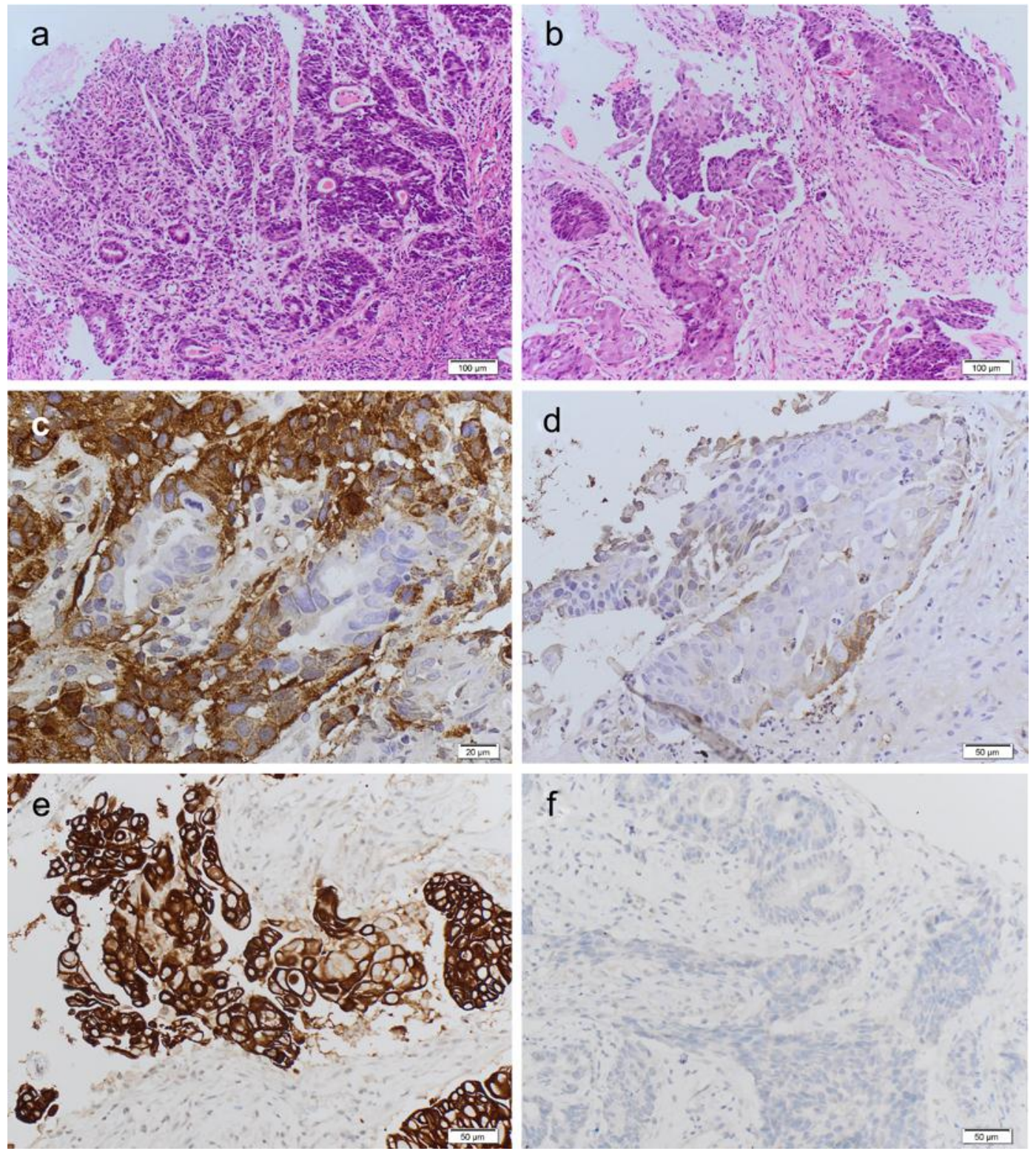

Fig. 2. Microscopic appearance of the MANEC tumour. a Moderately differentiated adenocarcinoma intimately admixed with a poorly differentiated neuroendocrine carcinoma. HE $\times 10$. b Squamous cell carcinoma component admixed with the NEC component. Squamous $\times 10$. c Positivity of the neuroendocrine component for chromogranin and negativity of the adenocarcinomatous component for chromogranin. Chromogranin $\times 20$. $\mathbf{d}$ Negativity of the squamous cell carcinoma for chromogranin and positivity of the NEC for chromogranin. Chromo-squamous $\times 20$. e Squamous cell differentiation as confirmed by the positivity for high molecular weight cytokeratins such as CK5. CK5-squamous $\times 20$. $f$ Negativity of both the adenocarcinomatous component and the NEC component for CK5 . CK5adeno-NEC. 


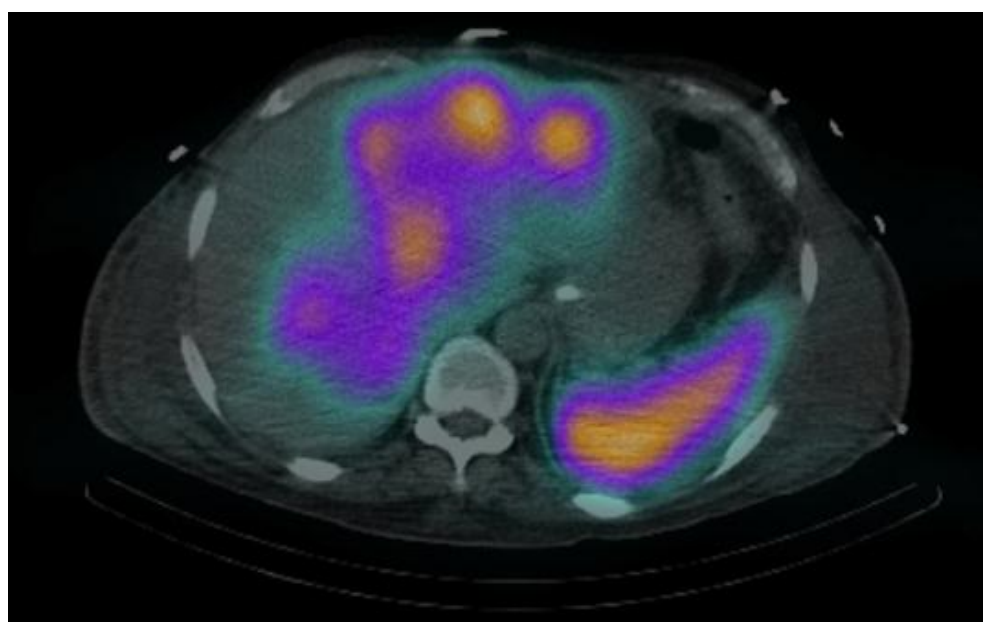

Fig. 3. Indium-111 Octreoscan SPECT-CT, liver and stomach uptake.

\section{References}

$\checkmark 1$ Solcia E, Bordi C, Creutzfeld W, et al: Histopathological classification of nonantral gastric endocrine growths in man. Digestion 1988;41:185-200.

2 Lewin KJ, Appelman HD: Tumors of the esophagus and stomach; in Rosai J, Sobin LH (eds): Atlas of Tumor Pathology, ser 3, fasc 18. Washington, Armed Forces Institute of Pathology, 1996, pp 351-352.

-3 Fujiyoshi Y, Kuhara H, Eimoto T: Composite glandular-endocrine cell carcinoma of the stomach. Report of two cases with goblet cell carcinoid component. Pathol Res Pract 2005;200:823-829.

4 Bosman FT, Carneiro F, Hruban RH, Theise ND: WHO Classification of Tumours of the Digestive System, ed 4. Lyon, IARC, 2010, pp 13-14.

-5 Haratake J, Horie A, Inoshita S: Gastric small cell carcinoma with squamous and neuroendocrine differentiation. Pathology 1992;24:116-120.

6 Shibuya H, Azumi N, Abe F: Gastric small-cell undifferentiated carcinoma with adeno and squamous cell carcinoma components. Acta Pathol Jpn 1985;35:473-480.

7 Nugent SL, Cunningham SC, Alexiev BA, et al: Composite signet-ring cell/neuroendocrine carcinoma of the stomach with a metastatic neuroendocrine carcinoma component: a better prognosis entity. Diagn Pathol 2007;2:43.

-8 Furlan D, Cerutti R, Genasetti A, et al: Microallelotyping defines the monoclonal or the polyclonal origin of mixed and collision endocrine-exocrine tumors of the gut. Lab Invest 2003;83:963-971.

-9 Kim KM, Kim MJ, Cho BK, et al: Genetic evidence for the multi-step progression of mixed glandularneuroendocrine gastric carcinomas. Virchows Arch 2002;440:85-93.

10 Kim TY, Chae HD: Composite neuroendocrine carcinoma with adenocarcinoma of the stomach misdiagnosed as a giant submucosal tumor. J Gastric Cancer 2011;11:126-130.

11 Volante M, Rindi G, Papotti M: The grey zone between pure (neuro)endocrine and non-(neuro)endocrine tumours: a comment on concepts and classification of mixed exocrine-endocrine neoplasms. Virchows Arch 2006;449:499-506.

12 Pavel M, Baudin E, Couvelard A., et al: ENETS consensus guidelines for the management of patients with liver and other distant metastases from neuroendocrine neoplasms of foregut, midgut, hindgut, and unknown primary. Neuroendocrinology 2012;95:157-176. 